\title{
Self-Assembly of a Library of Polyborate Chiral Anions for Asymmetric Catalytic Quinoline Reduction
}

\author{
Aman A. Desai, Yong Guan, Aaron L. Odom, Supriyo Majumder and William D. Wulff*, Department \\ of Chemistry. Michigan State University, East Lansing, Michigan 48824 \\ and
}

Mathew J. Vetticatt, Department of Chemistry, Binghamton University, Binghamton, NY 13902

\section{Email: wulff@chemistry.msu.edu}

\begin{abstract}
The 'template' polyborate BOROX catalysts are shown to mediate the asymmetric transfer hydrogenation of 2-quinolines. The rapid and simple generation of a large family of BOROX catalysts with significantly altered asymmetric pockets is described. A transition state model that explains the enantioselectivity is proposed.
\end{abstract}

Keywords: VANOL, BOROX catalyst, quinoline, Hantzsch ester, polyborate, boroxinate anion, asymmetric transfer hydrogenation

We have identified, by crystallographic and NMR studies, the active catalyst in our catalytic asymmetric aziridination reaction (AZ reaction). ${ }^{1}$ The catalyst is a polyborate chiral anion that contains a boroxine ring in which one of the borons is four coordinate producing a boroxinate core (Figure 1). ${ }^{2}$ These boroxinate or BOROX catalysts, are self-assembled by a substrate of the catalyst, which is an imine or amine in the case of the aziridination reaction (Scheme 1). ${ }^{3}$ The base induced assembly of the BOROX catalyst can be achieved from: 1) the ligand, water, and triphenylborate, ${ }^{3-5}$ 2) the ligand and triphenoxyboroxine ${ }^{1 b}$ and 3) the ligand, borane dimethysulfide complex, phenol and water. ${ }^{4,6}$ 


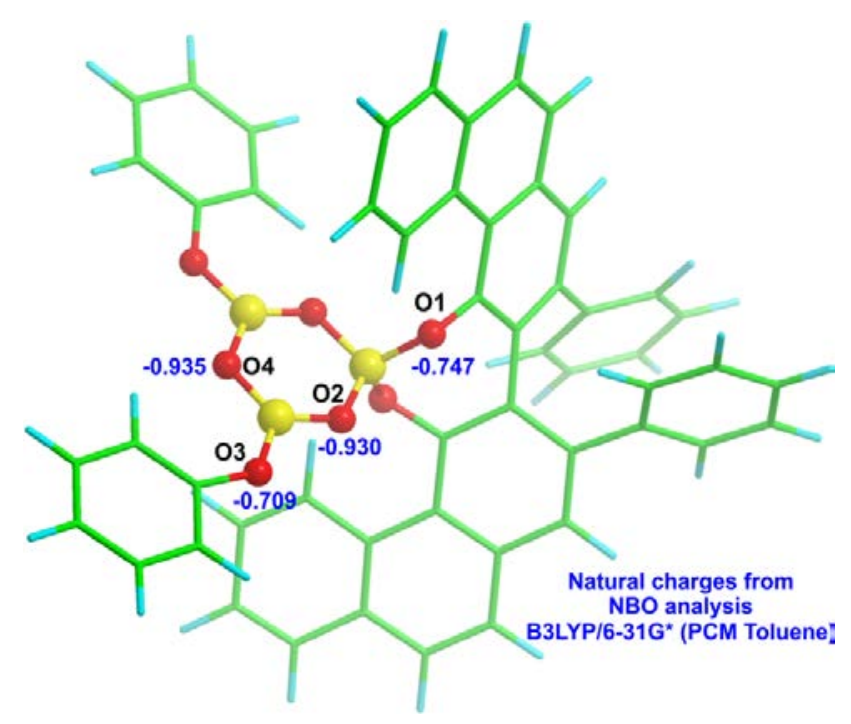

Figure 1. (S)-VAPOL-BOROX anion.

Theoretical studies of the AZ reaction have shed light on the mode of catalysis of this chiral polyborate Brønsted acid catalyst. ${ }^{1 \mathrm{c}, \mathrm{d}}$ In addition to rendering proton catalysis enantioselective, it was proposed that the VAPOL/VANOL-BOROX counterion also plays the role of orchestrating the orientation of the substrates at the key transition state via multiple H-bonding interactions with oxygens $\mathrm{O} 1, \mathrm{O} 2$ and $\mathrm{O} 3$ in the boroxinate core. ${ }^{1 \mathrm{c}, \mathrm{d}}$ In addition to aziridinations, the BOROX catalyst is also involved in an asymmetric catalytic aza-Cope rearrangement in the aminoallylation of aldehydes, ${ }^{7}$ in a catalytic asymmetric 3-componnent Ugi reaction ${ }^{8}$ and presumably in a heteroatom Diels-Alder reaction

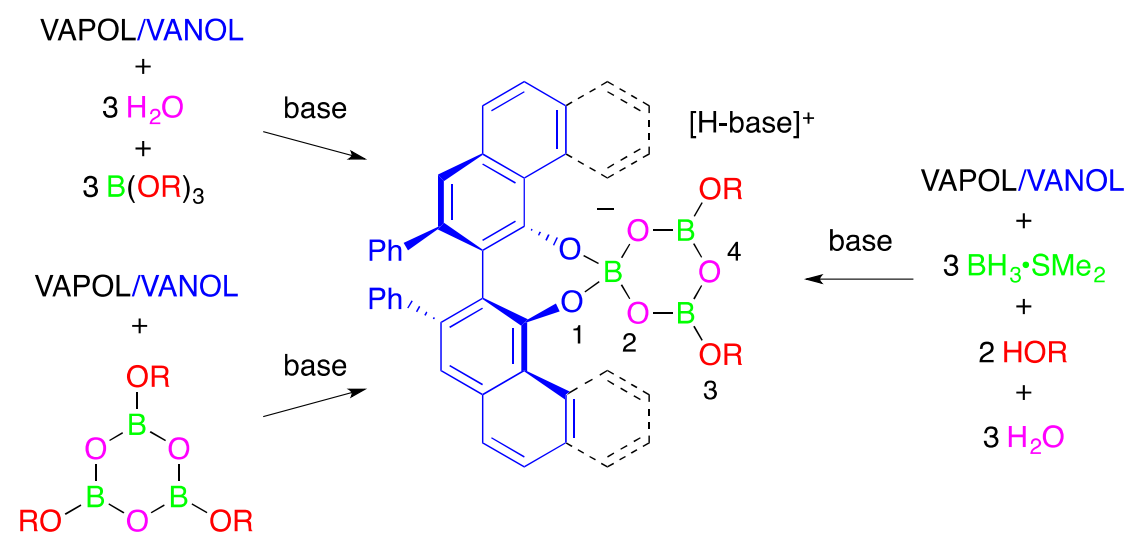

Scheme 1. Self-assembly of boroxinate (BOROX) catalyst. 
as well. ${ }^{9}$ Since the BOROX catalyst is self-assembled in-situ when the substrate is added, it thus becomes possible to generate a vast library of the BOROX catalysts on the spot by the incorporation of different alkoxy groups in the boroxinate core. ${ }^{10}$ Although a few borate esters are commercially available, a far greater avenue for maximizing diversity is to directly assemble the catalyst in the presence of a commercially available alcohol or phenol. ${ }^{8}$ Herein we report our efforts towards utilizing the diversity of this 'template' BOROX catalyst in the asymmetric transfer hydrogenation (ATH) of quinolines with Hantzsch's ester. ${ }^{11,12}$ In addition, we propose a transition state model that accounts for the experimental observations.

We expected the quinolines $\mathbf{1 a}$ and $\mathbf{1 b}$ chosen for the present study to smoothly self-assemble the

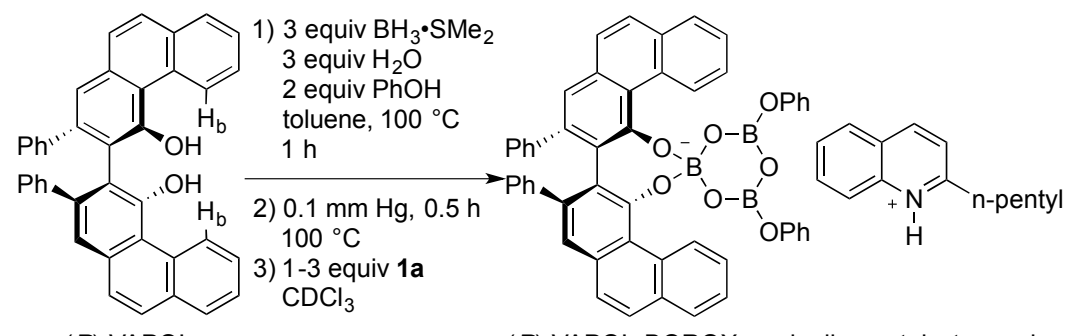

(R)-VAPOL

(R)-VAPOL-BOROX - quinoline catalyst complex
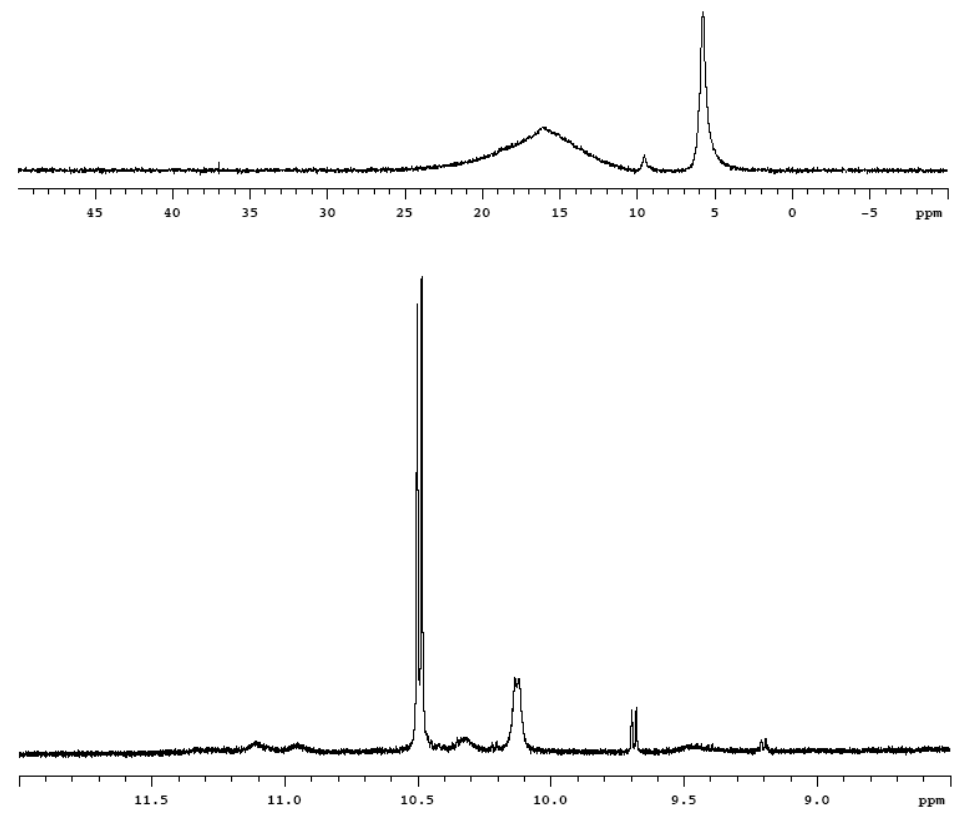

Figure 2. Preparation of (R)-VAPOL-BOROX-1a complex and its ${ }^{11} \mathrm{~B} \mathrm{NMR}\left(\mathrm{CDCl}_{3}, 160 \mathrm{MHz}\right)$ and ${ }^{1} \mathrm{H} \mathrm{NMR}\left(\mathrm{CDCl}_{3}, 500 \mathrm{MHz}\right)$.

polyborate BOROX catalysts, similar to the imines and other bases in our previous studies. ${ }^{1,3}$ The ${ }^{11} \mathrm{~B}$ and ${ }^{1} \mathrm{H}$ NMR spectra of the VAPOL-BOROX complex with 1a were distinctive (Figure 2). The two 3- 
coordinate borons appear as a broad peak at $15.97 \mathrm{ppm}$, and the 4-coordinate boron as a sharp peak at $5.76 \mathrm{ppm}$, with an integration of 2:1 respectively (not shown). The most distinctive peak in the ${ }^{1} \mathrm{H}$ NMR spectrum of the VAPOL-BOROX-1a complex is the bay region doublet at $10.49 \mathrm{ppm}\left(\mathrm{H}_{\mathrm{b}}\right.$ in VAPOL). These observations are in accord with those made previously for the BOROX catalysts in our aziridination studies. $^{1,3}$

Initial attempts towards developing the catalytic asymmetric transfer hydrogenation reaction utilized the 2-pentylquinoline substrate 1a and the Hantzsch ester $\mathbf{2 a}$ as the hydride transfer reagent (Table 1). The 2-pentylquinoline was prepared by a titanium mediated multi-component coupling of an aniline, an alkyne and a isonitrile. ${ }^{13}$ Whilst the VANOL-BOROX catalyst 4 afforded the tetrahydroquinoline product 3a with a 40:60 e.r. (Entry 1), the corresponding BOROX catalyst 5 prepared from the VAPOL ligand provided 3a in quantitative yield and with a promising e.r. of 86:14 (Entry 2). To optimize this protocol, different Hantzsch ester derivatives 2a-d were subsequently screened, albeit without any big improvements in the e.r. (entries 3-5). Additives were found to be deleterious in this reaction, and an extensive screen of 13 solvents established benzene as the solvent of choice. ${ }^{13}$ In addition, a number of substituted VANOL and VAPOL ligands were evaluated but none were superior to VAPOL. ${ }^{14}$ High yields and similar trends for the enantiomeric ratios were also observed for the ATH of 2-phenylquinoline $\mathbf{1 b}$ (entries 6-7). These initial results were promising and we decided to pursue them for our catalyst optimization studies.

The BOROX catalyst in most of our aziridination studies was generated from $\mathrm{B}(\mathrm{OPh})_{3}$ as the boron source, ${ }^{1,3,4}$ which was also the method in the preliminary screens in Table 1 . It was found in the present study that replacing $\mathrm{B}(\mathrm{OPh})_{3}$ with the combination of $\mathrm{BH}_{3} \bullet \mathrm{SMe}_{2}$ and phenol provided identical results (compare result with phenol in Scheme 2 with that in entry 2 of Table 1). That the BOROX polyborate catalyst could be successfully generated with a quinoline via the $\mathrm{BH}_{3} \bullet \mathrm{SMe}_{2}$ route then lent us the Table 1. Quinoline reduction with the VAPOL/VANOL-BOROX catalysts. ${ }^{\text {a }}$ 


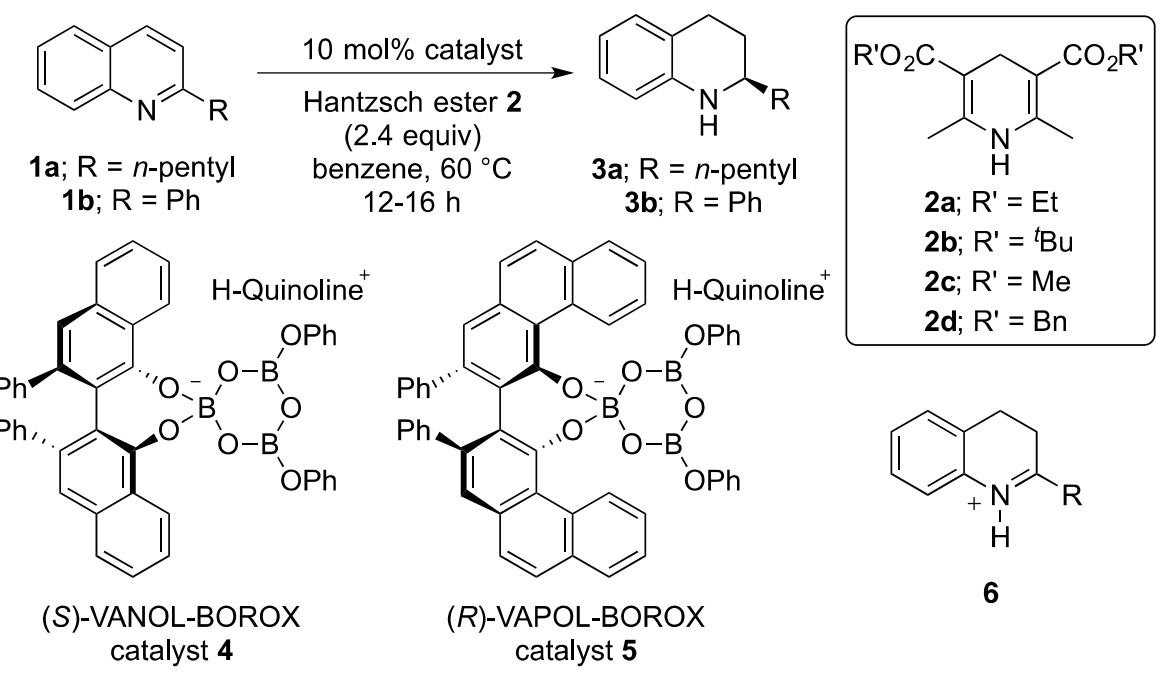

\begin{tabular}{ccccccc}
\hline Entry & $\mathbf{1}$ & $\mathbf{2}$ & Catalyst & $\mathbf{3}$ & ${\text { Yield-3 }(\%)^{b}}^{b}$ & e.r.-3 (\%) ${ }^{c}$ \\
\hline 1 & $\mathbf{1 a}$ & $\mathbf{2 a}$ & $\mathbf{4 a}$ & $\mathbf{3 a}$ & 88 & $40: 60$ \\
$2^{d}$ & $\mathbf{1 a}$ & $\mathbf{2 a}$ & $\mathbf{5 a}$ & $\mathbf{3 a}$ & $>99$ & $86: 14$ \\
& & & & & & \\
$3^{e}$ & $\mathbf{1 a}$ & $\mathbf{2 b}$ & $\mathbf{5 a}$ & $\mathbf{3 a}$ & $\mathrm{ND}^{f}$ & $72: 28$ \\
4 & $\mathbf{1 a}$ & $\mathbf{2 c}$ & $\mathbf{5 a}$ & $\mathbf{3 a}$ & $\mathrm{ND}^{f}$ & $88: 12$ \\
5 & $\mathbf{1 a}$ & $\mathbf{2 d}$ & $\mathbf{5 a}$ & $\mathbf{3 a}$ & $\mathrm{ND}^{f}$ & $88: 12$ \\
& & & & & & \\
6 & $\mathbf{1 b}$ & $\mathbf{2 a}$ & $\mathbf{4 b}$ & $\mathbf{3 b}$ & $>99$ & $32: 68$ \\
$7^{g}$ & $\mathbf{1 b}$ & $\mathbf{2 a}$ & $\mathbf{5 b}$ & $\mathbf{3 b}$ & $>99$ & $84: 16$ \\
\hline
\end{tabular}

${ }^{a}$ Unless otherwise specified, all reactions were run with $0.05 \mathrm{mmol}$ of $\mathbf{1 a}$ and $0.1 \mathrm{mmol}$ of $\mathbf{1 b}$. The catalyst was prepared by heating 1 equiv of ligand, 4 equiv of $\mathrm{B}(\mathrm{OPh})_{3}$, and 1 equiv of water in toluene at $80^{\circ} \mathrm{C}$ for $1 \mathrm{~h}$, followed by removing all volatiles under high vacuum $(0.1 \mathrm{~mm} \mathrm{Hg})$ at $80^{\circ} \mathrm{C}$ for $0.5 \mathrm{~h}$. Conversion $=100 \%$ (TLC). Reaction with (S)-VANOL-BOROX catalyst 4 gives ent-3. ${ }^{b}$ Isolated yield after column chromatography on regular silica gel. ${ }^{c}$ Determined by HPLC on isolated 3. ${ }^{d}$ Average of 3 runs. ${ }^{e}$ Average of 4 runs. ${ }^{\text {Product }} 3$ isolated by pipette column chromatography on regular silica gel, usually in $>99 \%$ isolated yield. ${ }^{g}$ Reaction time was $58 \mathrm{~h}$.

unique opportunity to generate, in a rapid manner, a large library of these BOROX catalysts 7 (Scheme 1) by simply incorporating different phenols and alcohols during the catalyst self-assembly process. Such a family of BOROX catalysts would offer significantly altered active site polyborate cores; and such a self-assembly process would automatically forgo the tedious synthesis normally associated with preparing catalyst derivatives. 
Scheme 2. Self-assembly of a family of BOROX catalysts (7).

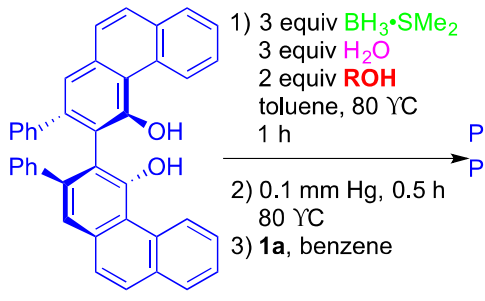

$(R)$-VAPOL

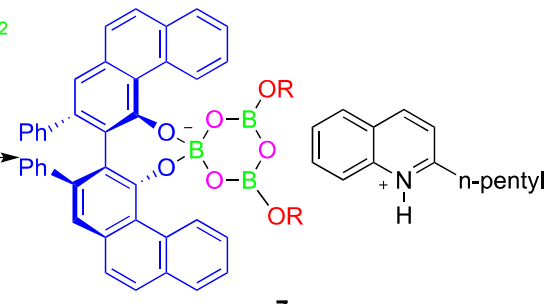

7

family of $(R)$-VAPOL-BOROX - quinoline catalyst complexes

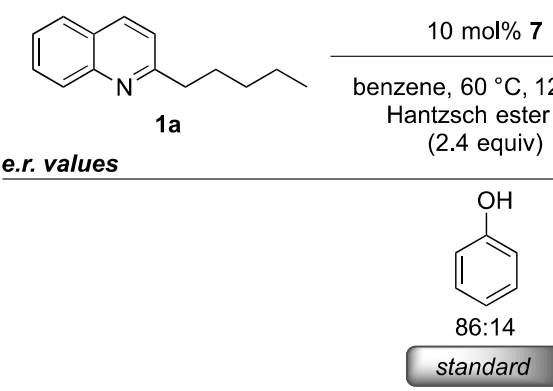<smiles>CCc1ccc(O)cc1</smiles>

$86: 14$<smiles>CC(C)(C)c1ccc(O)cc1</smiles>

$76: 24$ $\rightarrow$

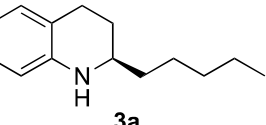

3a

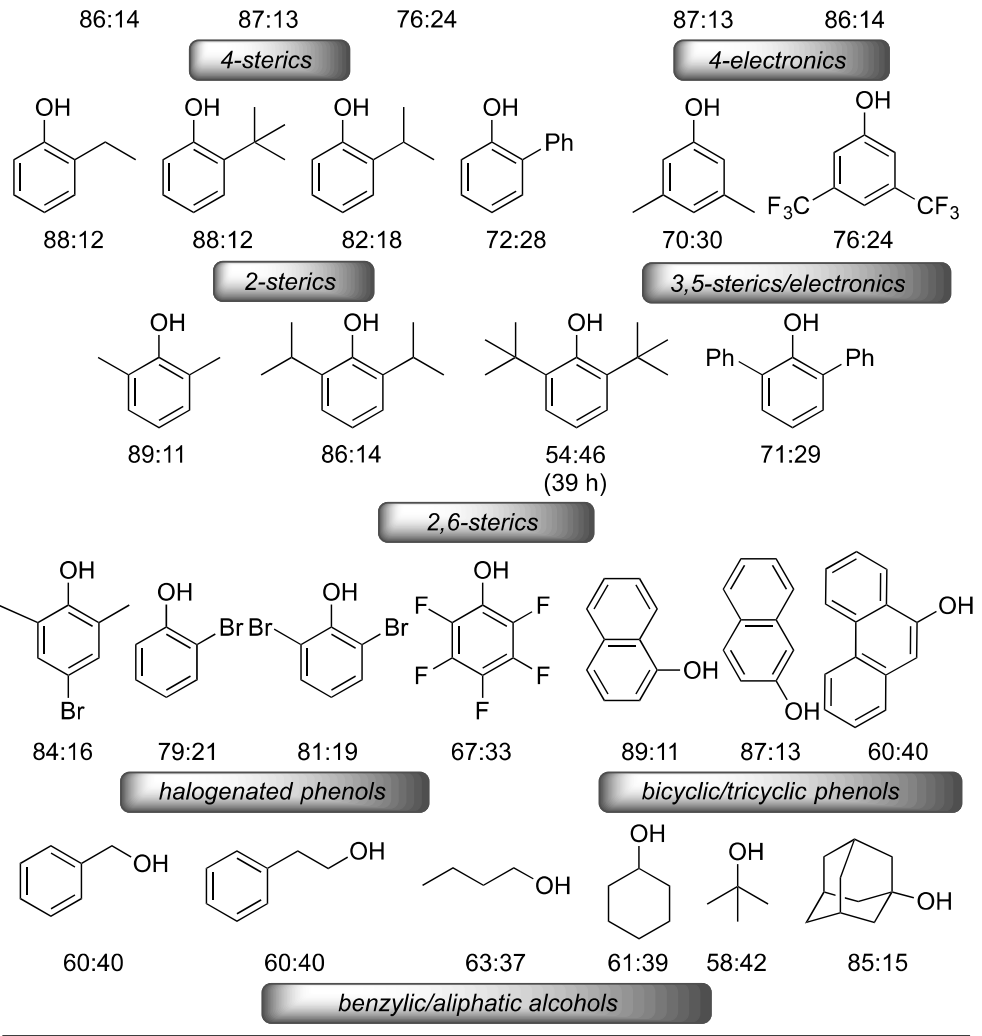

a All reactions were run at $0.05 \mathrm{mmol}$ scale of $1 \mathrm{a}$ and the conversion was $100 \%$ (TLC), The product $3 a$ was isolated (usually in $>99 \%$ yield) by column chromatography on silica gel.

A proof of principle for this concept has been established for the ATH reaction and is shown in Scheme $2 ;{ }^{15}$ a broad range of commercially available and sterically/electronically different phenols and 
alcohols were utilized to self-assemble a family of BOROX catalysts (7), which were subsequently evaluated for the ATH reaction of 2-pentylquinoline 1a. The results were gratifying in that the gamut of asymmetric inductions was observed, from an e.r. of 54:46 for the BOROX catalyst prepared from 2,6di-tert-butylphenol to an e.r. of 89:11 for that prepared from 2,6-dimethylphenol and 1-naphthol, thus establishing the importance of this region in the active site polyborate core of the BOROX catalyst.

The mechanism of the Hantzsch ester reduction of quinolines, mediated by a Brønsted acid catalyst, involves a two-step process where the enantiogenic step is the reduction of the dihydroquinolinium ion $\mathbf{6}$ (Table 1). ${ }^{12 a, 16}$ The hydride transfer to the dihydroquinoline $\mathbf{6}$, in the reaction of 1a and 2a catalyzed by the (R)-VAPOL-BOROX catalyst, was modeled using ONIOM(B3LYP/6$31 \mathrm{G}^{*}:$ AM1) calculations. ${ }^{17}$ The main goal of this theoretical study was to understand the catalystsubstrate interactions that stabilize this transition state. The reported relative energies are from freeenergy estimates based on the unscaled harmonic frequencies obtained from the ONIOM calculations.

The lowest energy transition structures leading to the $R$ enantiomer (TS1) and the $S$ enantiomer (TS2) of 3a are shown in Figure 3. Transition structures TS1 and TS2 correspond to hydride transfer from 2a to the iminium carbon of the O3-bound dihydroquinoline 6 (See Figure 1 for numbering of boroxinate oxygen atoms). The orientation of $\mathbf{2 a}$ as it transfers the hydride is determined by a strong $(\sim 1.9 \AA) \mathrm{H}$-bond interaction between the $\mathrm{N}-\mathrm{H}$ of $\mathbf{2 a}$ and $\mathrm{O} 1$ of the catalyst core. ${ }^{18}$ The key difference between the two transition structures is that hydride transfer occurs to the pro- $R$ face of $\mathbf{6}$ in TS1 and to the pro-S face in TS2. Transition structure TS1 is $0.4 \mathrm{kcal} / \mathrm{mol}$ lower in energy than TS2. This prediction, though in the correct direction, is an underestimation of the experimental enantioselectivity (experimental difference is $\sim 1.1 \mathrm{kcal} / \mathrm{mol}$ for $86: 14$ e.r. at $60^{\circ} \mathrm{C}$ ). This discrepancy can possibly be attributed to inaccuracies arising as a consequence of (a) the size of the computational system, (b) the level of theory used and (c) not including a solvent model in our calculations. A close examination of TS1 and TS2 also leads to a more interesting explanation for this discrepancy - the hydride is 'more 
transferred' in TS1 and this transition structure is characterized by a larger imaginary frequency (-985) as compared to a smaller value (-707) for the 'less transferred' hydride in TS2. The magnitude of the imaginary frequency is inversely proportional to the width of the reaction barrier. This suggests that the $0.4 \mathrm{kcal} / \mathrm{mol}$ preference for TS1 over TS2 is likely an underestimation of the relative rates of formation of the two enantiomers - hydrogen tunneling would make a larger contribution to the rate of the reaction proceeding via TS1 (narrow barrier) as compared to TS2 (wider barrier).

Figure 3. Key transition structures for hydride transfer to O3-bound 6. All distances are in angstroms. $\left(\mathrm{E}=\mathrm{CO}_{2} \mathrm{Et}\right)$

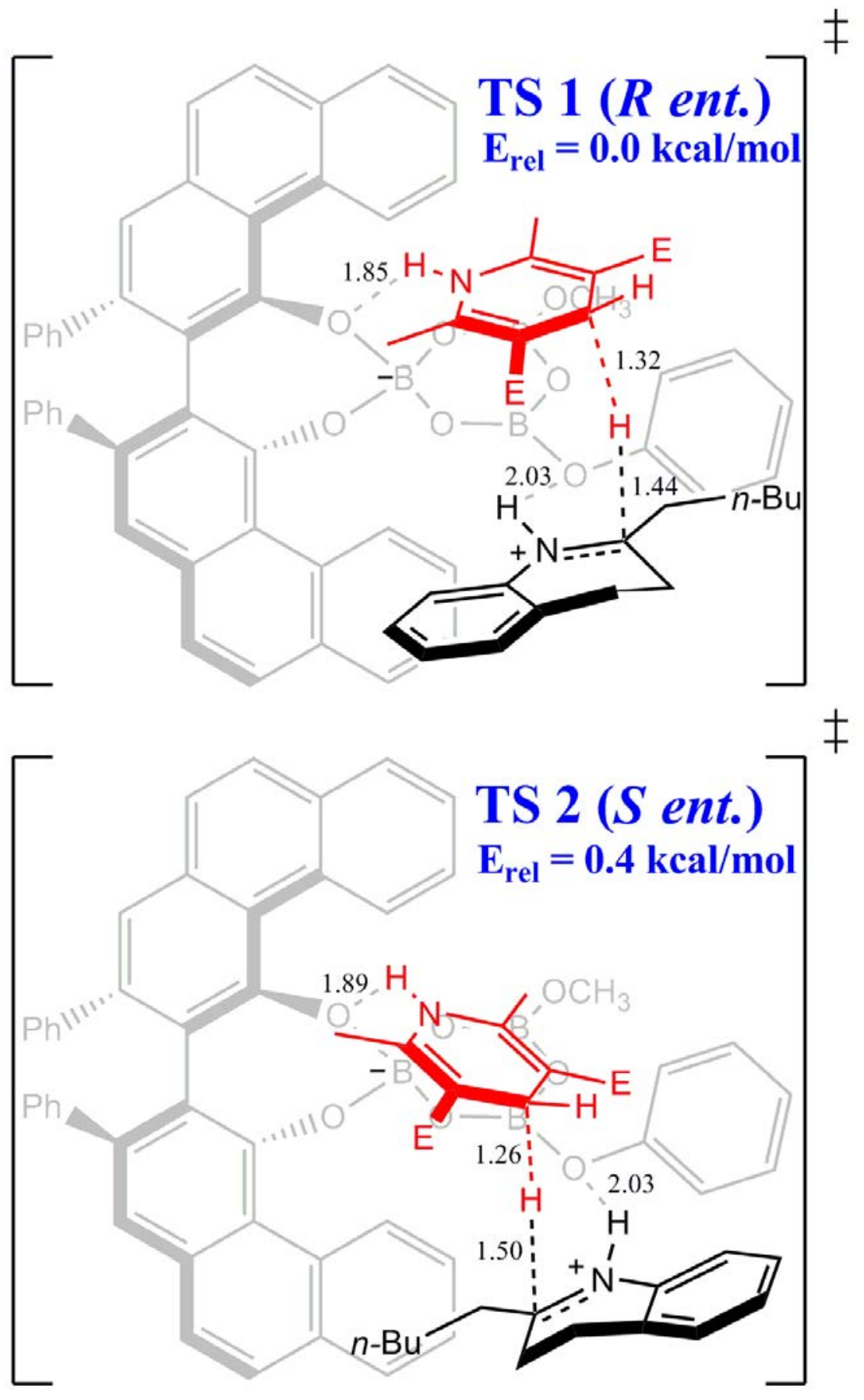


A pair of transition structures was also located for the reversed H-bonding scenario, with $\mathbf{6}$ bound to $\mathrm{O} 1$ and $2 \mathrm{a}$ bound to $\mathrm{O} 3$. These structures were found to be 4.5 ( $R$ ent.) and 4.8 (S ent.) $\mathrm{kcal} / \mathrm{mol}$ higher in energy than TS1 (See Supporting Information). Finally, the importance of both Hbonding interactions in stabilizing the transition state was illustrated when a pair of transition structures corresponding to hydride transfer to the pro- $R$ and pro-S faces of the O2-bound 6 was located. These structures, lacking the second H-bonding interaction between the catalyst and 2a, were found to be 16.4 (R ent.) and 9.0 (S ent.) kcal/mol higher in energy than TS1 (See Supporting Information).

In conclusion, we have shown that our 'template' polyborate BOROX catalyst catalyzes the asymmetric transfer hydrogenation of 2-quinolines with reasonable levels of enantioselectivity. We have also established a simple protocol for generating a large library of these BOROX catalysts with different sterics and electronics at the active site polyborate core. The lowest energy transition structures (TS1 and TS2) leading to the two enantiomeric products have been identified. The transition state model is consistent with the one proposed for our aziridination reaction mediated by the same catalyst, ${ }^{1 \mathrm{~d}, \mathrm{~d}}$ where the chiral counterion also offers H-bonding sites to orient the substrates at the stereochemistry determining transition state. Attempts to further improve the asymmetric inductions of the present study are ongoing, as are those to apply this novel library of polyborate BOROX catalysts for other asymmetric reactions.

Acknowledgment. Support provided by the National Institute of General Medical Sciences (GM094478). ALO and SM thank the National Science Foundation (CHE-1012537) for financial support.

References:

[1] (a) Hu, G.; Huang, L.; Huang, R. H.; Wulff, W. D. J. Am. Chem. Soc. 2009, 131, 15615-15617. (b) Hu, G.; Gupta, A. K.; Huang, R. H.; Mukherjee, M.; Wulff, W. D. J. Am. Chem. Soc. 2010, 132, 
14669-14675. (c) Vetticatt, M. J.; Desai, A. A.; Wulff, W. D. J. Am. Chem. Soc. 2010, 132, 1310413107. (d) Vetticatt, M. J.; Desai, A. A.; Wulff, W. D. J. Org. Chem. 2013, 78, 5142.

[2] For a review of organoboron acids in catalysis, see: Dimitrijevic, E.; Taylor, M. S. ACS Catal. 2013, 3, 945.

[3] Gupta, A. K.; Mukherjee, M.; Hu, G.; Wulff, W. D. J. Org. Chem. 2012, 77, 7932-7944.

[4] Zhang, Y.; Desai, A.; Lu, Z.; Hu, G.; Ding, Z.; Wulff, W. D. Chem. Eur. J. 2008, 3785-3803.

[5] Commercial $\mathrm{B}(\mathrm{OPh})_{3}$ contains enough water that its addition is usually not needed.

[6] The BOROX catalyst has also been made from the ligand, boric acid and phenol: Wang, Z.; Li, F.; Zhao, L.; He, A.; Chen, F.; Zheng, C. Tetrahedron 2011, 67, 9199-9203.

[7] Ren, H.; Wulff, W. D. J. Am. Chem. Soc. 2011, 133, 5656-5659.

[8] Zhao, W.; Huang, L.; Guan, Y.; Wulff, W. D. Angew. Chem. Int. Ed. 2014, 53, 3436.

[9] Newmann, C. A.; Antilla, J. C.; Chen, P.; Wulff, W. D. J. Am. Chem. Soc. 2007, 129, 72167217.

[10] For reviews on other types of self-assembled catalysts, see: (a) Gasparini, G.; Molin, M. D.; Prins, L. J. Eur. J. Org. Chem. 2010, 2429. (b) Meeusissen, J.; Reek, J. N. H. Nature Chem. 2010, 2, 615. (c) Briere, J.-F.; Oudeyer, S.; Dalla, V.; Leavacher, V., Chem. Soc. Rev. 2012, 41, 1696. (d) Brak, K.; Jacobsen, E. N. Angew. Chem. Int. Ed. 2013, 52, 534. (e) Raynal, M.; Ballester, P.; Vidal-Ferran, A.; van Leeuwen, P. W. N. M. Chem. Soc. Rev. 2014, 43, 1660. For other types of self-assembled boron catalysts, see footnote 2 and: (f) Barnett, D. S.; Schaus, S. E. Org. Lett. 2011, 13, 4020. (g) Hashimoto, T.; Galvez, A. O.; Maruoka, K. J. Am. Chem. Soc. 2013, 135, 17667.

[11] For a review of transfer hydrogenation with Hantzsch esters, see: Zhang, C.; You, S. L. Chem. Soc, Rev. 2012, 41, 2498.

[12] For other catalytic asymmetric transfer hydrogenation of quinolines with Hantzsch's ester, see (a) Rueping, M.; Antonchick, A. P.; Theissmann, T. Angew. Chem. Int. Ed. 2006, 45, 3683-3686. (b) Guo, Q.-S.; Du, D.-M.; Xu, J. Angew. Chem. Int. Ed. 2008, 47, 759-762. (c) Rueping, M.; Theissmann, T.; Raja, S.; Bats, J. W. Adv. Synth. Catal. 2008, 350, 1001. (d) Rueping, M.; Sugiono, E.; Steck, A.; Theissmann, T. Adv. Synth. Catal. 2010, 352, 281. (e) Rueping, M.; Theissmann, T. Chem. Sci. 2010, 1, 473. (f) Rueping, M.; Hubener, L. Synlett 2011, 1243. (g) Rueping, M.; Theissmann, T.; Stoeckel, M.; Antonchick, Org. Biomol. Chem. 2011, 9, 6844. (h) Ren, L.; Lei, T.; Ye, J.-X.; Gong, L.-Z. Angew. Chem. Int. Ed. 2012, 51, 771. (i) Liu, X.-Y.; Xiao, Y.-P.; Siu, F.-M.; Ni, L.-C.; Chen, Y.; Wang, L.; Che, C.-M. Org. Biomol. Chem. 2012, 10, 7208. (j) Tu, X.-F.; Gong, L.-Z. Angew. Chem. Int. Ed. 2012, 51, 11346. (k) Liao, H.-H.; Hsiao, C.-C.; Sugiono, E.; Rueping, M.; Chem. Commun. 2013, 49, 7953. (l) Sugiono, E.; Rueping, M. Beilstein J. Org. Chem. 2013, 9, 2457. (m) Patil, N. T.; Raut, V. S.; Tella, R. B. Chem. Commun. 2013, 49, 570. (n) Wang, S.-G.; Zhang, W.; You, S.-L. Org. Lett. 2013, 15, 1488. (o) Lackner, A. D.; Samant, A. V.; Toste, F. D. J. Am. Chem. Soc. 2013, 135, 14090. (p) Ferry, A.; Stemper, J.; Marinetti, A.; Voituriez, A.; Guinchard, X. Eur. J. Org. Chem. 2014, 188. (q) Chen, M.-W.; Cai, X.-F.; Chen, Z.-P.; Shi, L.; Zhou, Y.-G. Chem. Commun. 2014, 50, 12526. (r) Cai, X.-F.; Guo, R.N.; Feng, G.-S.; Wu, B.; Xhou, Y.-G. Org. Lett. 2014, 16, 2680. (s) Aillerie, A.; de Talance, V. L.; Moncomble, A.; Bousquet, T.; Pelinski, L. Org. Lett. 2014, 16, 2982. (t) Guo, R.-N.; Chen, Z.-P.; Cai, X.-F.; Zhou, Y.-G. Synthesis 2014, 2751.

[13] (a) Majumder, S.; Gipson, K. R.; Odom, A. L. Org. Lett. 2009, 11, 4720-4723. (b) Cao, C.; Shi, Y.; Odom, A. L. J. Am. Chem. Soc. 2003, 125, 2880-2881. 
[14] See Supporting Information for complete details.

[15] A proof of principle for this concept has also been established for the Ugi reaction (reference 8).

[16] Marcelli, T.; Hammar, P.; Himo, F. Chem. Eur. J. 2008, 14, 8562.

[17] Svensson, M.; Humbel, S.; Morokuma, K. J. Chem. Phys. 1996, 105, 3654-3661. (b) Vreven, T.; Morokuma, K. J. Comput. Chem. 2000, 21, 1419-1432. (c) Dapprich, S.; Koma'romi, I.; Byun, K. S.; Morokuma, K.; Frisch, M. J. J. Mol. Struct. 1999, 461, 1-21. See Supporting Information for division of layers and other calculational details. We have not attempted to explain the effect of varying the phenols and alcohols on the enantioselectivity since in going from 89:11 e.r. (best) to 54:46 e.r. (worst), the change in the relative energies between the competing transition states is within the error of the calculational method used.

[18] For a similar TS model in a related reaction, see: Simon, L.; Goodman, J. M. J. Am. Chem. Soc. 2008, 130, 8741-8747.

Graphical Abstract: 


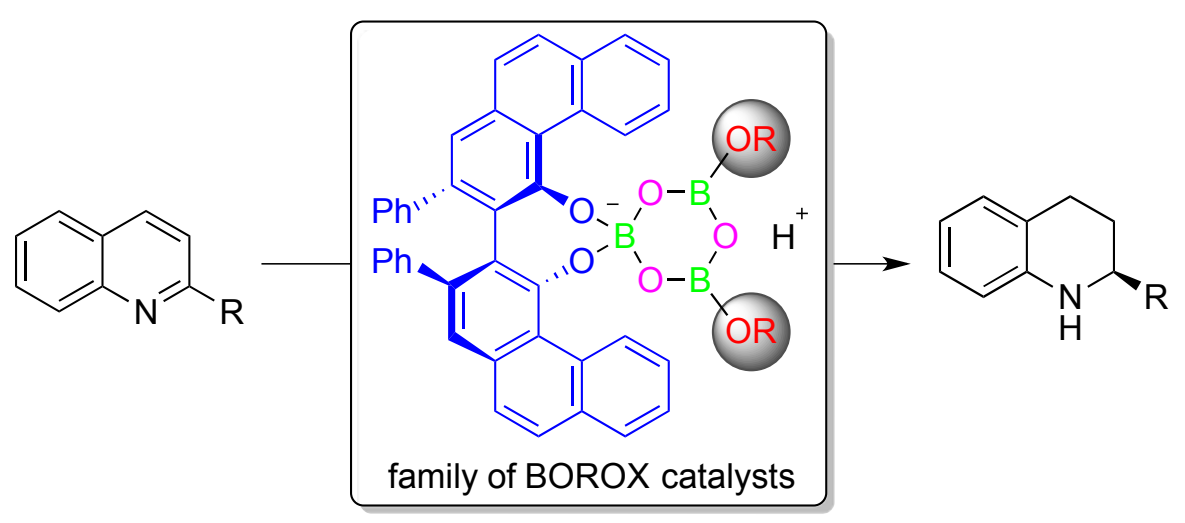

